\title{
Role of a non-canonical splice variant of the Helios gene in the differentiation of acute lymphoblastic leukemic $\mathbf{T}$ cells
}

\author{
YINGHUI LI ${ }^{1 *}$, YANHUA LIU ${ }^{1 *}$, CAN LIU $^{1}$, FENGYONG LIU ${ }^{2}$, DAOLEI DOU ${ }^{3}$, \\ WENJIE ZHENG ${ }^{4}$, WEI LIU ${ }^{4}$ and FEIFEI LIU ${ }^{1}$ \\ ${ }^{1}$ Department of Immunology, School of Medicine, Nankai University, Tianjin 300071; \\ ${ }^{2}$ Department of Pathophysiology, School of Basic Medical Sciences, Tianjin Medical University, Tianjin 300070; \\ ${ }^{3}$ Department of Experimental Facility, State Key Laboratory of Medical Chemical Biology, Tianjin 300071; ${ }^{4}$ Technical \\ Center for Safety of Industrial Products, Tianjin Entry-Exit Inspection and Quarantine Bureau, Tianjin 300308, P.R. China
}

Received August 13, 2017; Accepted February 21, 2018

DOI: $10.3892 / \mathrm{ol} .2018 .8214$

\begin{abstract}
T-cell acute lymphoblastic leukemia is a hematopoietic malignant disease, which arises from a genetic defect in the T-cell maturation signaling pathway. As a result, it is necessary to identify the molecules that impact T-cell development and control lymphoid-lineage malignancy. The present study utilized Jurkat $\mathrm{T}$ lymphoblastic cells as a well-established approach for the investigation into the function of the non-canonical alternative splice variant of Helios for the in vitro study of T-cell differentiation and leukemogenesis. In the present study, the Jurkat T-cell lines with stable overexpression of the wild-type (Helios-1) or the non-canonical short isoform (Helios- $\Delta 326-1431$ ), were established. RNA microarray, reverse transcription-quantitative polymerase chain reaction and flow cytometry were used to assess changes in the gene expression profiles and to monitor the cell surface markers during T-cell differentiation. Multiple genes associated with T-cell differentiation and leukemogenesis were identified as being either activated or suppressed. In addition, the results indicated that the stable overexpression of the Helios isoforms stimulated the differentiation pathway of the T-lineage lymphoblastic cells. Therefore, these results suggest that full-length Helios-1 has a tumor suppressor-like and immunomodulatory role, in contrast to the oncogenic function of the non-canonical short isoform Helios- $\Delta 326-1431$.
\end{abstract}

Correspondence to: Dr Feifei Liu, Department of Immunology, School of Medicine, Nankai University, 94 Weijin Road, Nankai, Tianjin 300071, P.R. China

E-mail: liufeifei@nankai.edu.cn

Dr Wei Liu, Technical Center for Safety of Industrial Products, Tianjin Entry-Exit Inspection and Quarantine Bureau, 2 Dong Wu Road, Tianjin 300308, P.R. China

E-mail: weiliutj@163.com

${ }^{*}$ Contributed equally

Key words: Ikaros, transcription factor, T-cell leukemia, Helios

\section{Introduction}

The expressions of the distinct transcription factors, target genes, and cell surface molecules dictate the lineage commitment and differentiation of $\mathrm{T}$ lymphocytes. The transcription factors, such as Notch1, E2A, Id proteins, Ikaros, and PU.1 perform definitive functions in the T-cell differentiation (1). Along the pathway of commitment and maturation, the early T-cell precursors upregulate the expressions of the transcription factors, such as Notch1, Ikaros, GATA3, and Runx1. However, they downregulate others, such as C/EBPa, Lmo2, GATA2, and PU.1 $(2,3)$. The expression patterns of the transcription factors are fundamental to the lineage commitment, specification, differentiation, and survival of the T-cells. Significantly, these regulatory factors which control normal development are frequently disturbed, and also implicated in T-cell transformations and leukemia (2).

Helios (Ikzf2), which is a zinc-finger DNA binding transcription factor and a key regulator of T-lineage differentiation, is a prime example $(4,5)$. Helios belongs to the Ikaros transcription factor family, and shares a common structure, which is characterized by two zinc finger domains, an N-terminal DNA-binding domain (core motif GGGAA), and a C-terminal dimerization domain $(4,6)$. Helios is specifically expressed in the T-cell lineage from the early stages of development. Furthermore, it has been shown that Helios is selectively induced by $>10$-fold in thymus-derived Foxp $3^{+}$ regulatory $\mathrm{T}$ (Treg) cells, and also regulates the differentiation of $\mathrm{T}$ helper cells and production of cytokines $(7,8)$. In addition, Helios augments the activation of Foxp3 by directly binding to the Foxp 3 promoter (9). Previous research studies have demonstrated that Helios interacts with the nucleosome remodeling and histone deacetylase (NuRD) complex, which suggests that Helios plays a pivotal role in chromatin remodeling, as well as the expression of target genes (10).

It is known that Helios controls lymphopoiesis and leukemogenesis (6,11-14). Recently we and other researchers identified multiple novel short isoforms of Helios which were overexpressed in patients with T-cell acute lymphoblastic leukemia, and demonstrated their dominant-negative function $(6,11)$. The peripheral blood mononuclear cells (PBMCs) 
of some of the T-cell leukemia patients expressed various short protein isoforms $(<55 \mathrm{kDa})$, which were not detected in healthy PBMCs. We found that one of these isoforms (Helios- $\Delta 326-1431,475$ bp) lacks part of exon 3, all of exons 4 to 6 , and embodies a nonsense mutation in exon 7 (6). Also, it has been determined that this novel short isoform lacks four N-terminal zinc fingers, which suggests that it is a putative dominant-negative isoform for the Ikaros gene family members (6). Therefore, the alternative splicing of Helios variants is possibly activated during leukemogenesis, and supports the role of a non-canonical isoform as an acute T-cell leukemic-type gene (ATL). These speculations have led to increased interest in the potential role of Helios in acute T-cell leukemia. However, the essential functions of the ATL-type Helios isoform have yet to be fully elucidated.

In this study, the ectopic overexpressions of the wild-type Helios-1, non-canonical short isoform Helios- $\Delta 326-1431$, and control in the Jurkat cells were utilized as the routine in vitro model of the T-cell acute lymphoblastic leukemia (T-ALL) for the purpose of investigating the function of Helios isoforms in the expression of important genes which are changed during T-cell development. This study examined in detail the modulated gene expression pattern using microarray. The results showed that the Helios isoforms regulated the transcriptional output of the target genes, as well as the epigenetic remodelers involved in leukocyte proliferation, cell cycle arrest, and growth. In addition, the Jurkat cells consisted of undifferentiated $\mathrm{T}$ lymphoblasts (15). The impact of the overexpression of the Helios isoforms on the differentiation of the Jurkat cells was also examined.

\section{Materials and methods}

Cell culture. In this study, the Jurkat cell line was purchased from the American Type Culture Collection (http://www.ATCC.com), and cultured as suggested by the manufacturer. The Jurkat T-cell lines with stable overexpression of wild-type Helios-1, non-canonical short isoform Helios- $\Delta 326-1431$, and control were established with lentiviral pLV-EF1 $\alpha$-MCS-IRES-Bsd expression vector (6). After selection by blasticidin $(2 \mu \mathrm{g} / \mathrm{ml})$ and confirmation by westernblot (6), the Jurkat cell lines with stable expression of Helios variants were maintained in RPMI-1640 medium (Gibco; Thermo Fisher Scientific, Inc., Waltham, MA, USA) which contained the following: $10 \%$ fetal calf serum, $\mathrm{NaHCO} 3$ $1.5 \mathrm{~g} / \mathrm{l}$, glucose $2.5 \mathrm{~g} / \mathrm{l}$, sodium pyruvate $0.11 \mathrm{~g} / 1$, and $50 \mathrm{U} / \mathrm{ml}$ penicillin and $50 \mathrm{~g} / \mathrm{ml}$ streptomycin at $37^{\circ} \mathrm{C}$ in $5 \% \mathrm{CO}_{2}(6)$. The 293T cells were cultured in DMEM medium containing $10 \%$ FCS.

mRNA extraction, reverse transcription and quantitative PCR assays. The total RNA was extracted from the harvested Jurkat cell lines by using $1 \mathrm{ml}$ of TRIzol reagent (Invitrogen; Thermo Fisher Scientific, Inc.) per 5x10 6 cells. The first-strand cDNA was generated from $2 \mathrm{mg}$ of the total RNA with the cDNA synthesis kit (Promega, Madison WI). The amplification of the Helios target transcripts was carried out with GoTaq qPCR master mix (Promega Corporation, Madison, WI, USA) in an ABI Prism 7000 analyzer (Applied Biosystems; Thermo Fisher Scientific, Inc.). The data were normalized using endogenous $\beta$-actin and GAPDH controls. The fold-changes of the expression pattern were calculated using a $\Delta \Delta \mathrm{Cq}$ method according to the instructions (Applied Biosystems). The nucleotide sequences for the primers were as follows: FOXN2 forward (F): CATCCAGGTCTAGCG TGTCT, reverse (R): TGCATAGCCACTGTCTCCAA; RUNX3 (F): CCCCTCCGTTCCTAACTGTT, (R) CCC TGCCAAGAGAACAGAGA; WNT3 (F): TCCATGCAG TTCCCAAGGAT, (R) TGAAATCCATGTGCCTCCCT; MLLT4 (F): CACATCGTGGACATGCTGAG, (R) CATCAT CGTCCTCCTCCTCC; MAST3 (F): CACCTCCCGCTA CTTCCTAG, (R) AGTCTTAATGCCTTGGCCCT; MSX2 (F): ATATGAGCCCTACCACCTGC, (R) GCTTTTCCA GTTCTGCCTCC; TP53 (F): TGGCCATCTACAAGCAGT CA, (R) GGTACAGTCAGAGCCAACCT; CD79A (F): CTT CCCTCTAAACTGCCCCA, (R) CACTAAGTGGCCCTG ACAGA; NDFIP2 (F): GTTTTATCCCGTGCCACCTC, (R) GCTGGTCTGCATCACTGAAG; CD40LG (F): TCA AATTGCGGCACATGTCA, (R) TGACTCGAAGCTTCC CGATT; SUMO1 (F): CTTCAACTGAGGACTTGGGG, (R) TCAGCAATTCTCTGACCCTCA; NOTCH2NL (F): AAC ATCGAGACCCCTGTGAG, (R) ATTCAGGCAAGGTCG AGACA; TCEAL8 (F): TCGAGGTGAGGGAAGAGAGA, (R) CTTCTGCCTCCTGTGGTACA; ZNF593 (F): CTT GGATGAGATTCACCGCG, (R) AAGTGGGTCTTCAGG TTGGT; NUDC (F): TTTCAGCCACCACAATCAGC, (R) AGCCTCTCTGCCTCTTCATC; CBX8 (F): GAAATG GAAGGGATGGTCGC, (R) GAGGAAGGTTTTGGGCTT GG; HCST (F): TTGCTACTTCTCTGCTCCCC, (R) ATC CGGAACAAGAGCCTGAA; TAB1 (F): GCAGAGCCA GAAATCCATGG, (R) GCTTGGCAAACTCAGTGTCA; NKAP (F): ATGGCCATGCTCTGTTACCT, (R) AGG GCTCTCTTCTCATCAGC; TGFA (F): GAAGCCACA AAGCCGGTAAA, (R) ATACTTACCGAGGGCTCACG; TNFSF9 (F): GGCCCAAAATGTTCTGCTGA, (R) CAA GTGAAACGGAGCCTGAG; MSX2 (F): ATATGAGCC CTACCACCTGC, (R) GCTTTTCCAGTTCTGCCTCC; HOXD11 (F): GGCTACGCTCCCTACTACG, (R) GTAGAA CTGGTCGAAGCCCT; HEYL (F): ATGCAAGCCAGG AAGAAACG, (R) AGAATCCTGTCCCACCAGTG; DGKQ (F): CACGTCTCCCTGTTTGTTGG, (R) CCATGTCCT TCAGCAGCATG.

Flow cytometry. In this study, FITC-conjugated antibodies against human CD3 (clone UCHT1), CD8a (RPA-T8), CD25 (M-A251), CD7 (M-T701), and the PE-conjugated antibody against human CD4 (RPA-T4) (BioLegend, Inc., San Diego, CA, USA) were used. Also, mouse IgG1 antibody was used as the isotype control. Following the labeling, the Jurkat cell lines were washed and suspended in an ice-cold staining media (phosphate buffer saline containing 5\% FBS, and $100 \mathrm{U} / \mathrm{ml}$ penicillin/streptomycin). The samples were processed in a FACS LSRFortessa (BD Biosciences, Franklin Lakes, NJ, USA), and the data were analyzed using Flowjo software (Tree Star, Inc., Ashland, OR, USA).

Gene-expression microarray and pathway analysis. The RNA was isolated from the Jurkat cell lines with stable expressions of the Helios isoforms. Gene expression microarray was performed with GeneChip human gene 2.0 ST array 
(Affymetrix; Thermo Fisher Scientific, Inc.). The details of the methods of the microarray analysis were previously described (16). The gene chip data were deposited into the Gene Expression Omnibus (GEO) database with the accession number GSE92416.

Statistical analysis. Statistical differences were determined using the unpaired Student's t-test and Ordinary one-way ANOVA with PRISM software (version 6.0c; GraphPad Software, Inc., La Jolla, CA, USA) for P-values. Multiple comparisons between the groups were performed using Tukey's HSD test. $\mathrm{P}<0.05$ was considered to indicate a statistically significant difference.

\section{Results}

Modulation of target gene expression by Helios-1. We established the Jurkat T-cell lines with stable overexpression of wild-type Helios-1, non-canonical short isoform Helios- $\Delta 326-1431$, or control by lentiviral transduction in our recent study (6). We then compared the microarray data for transcriptional analysis of the Jurkat cells with stable overexpression of full-length Helios-1 and mock transfected control (Fig. 1A). With a $>1.5$-fold cut-off, the analysis revealed that the genes encoding tumor-suppressors RUNX3 (1.639-fold) and TP53 (0.622) were deregulated in the Helios-1 cells. In addition, the expressions of the genes WNT3 (1.748-fold), FOXN2 (1.565), MLLT4 (1.715), TGFa (1.700), and TGFbR2 (0.607), which were involved in the regulation of human T-cell leukemia, were found to be deregulated in the Helios-1 Jurkat cells when compared to the control (Table I). The genes encoding lymphoid-lineage cell markers, such as CD7 (1.660-fold), CCL1 (1.599), CXCR3 (2.098), CD79a (0.517), and CD40 ligand (0.548), were determined to display altered expression patterns. The genes encoding innate immunity molecules, such as TLR3 (1.581) and LRRC37 (0.528), were also found to be modulated when compared to the control cell line.

The genome-wide transcriptional analysis of the Helios-1 and mock transfected Jurkat T-cell lines were used to compare and identify the potential pathways which may have the ability to support the aberrant growth properties of the mutant population (6). The group of genes which were deregulated in the Helios-1 T-cells indicated considerable enrichment for the genes which were involved in the pathways of the lymphocyte differentiation, lymphocyte proliferation, and regulation of cell growth $(\mathrm{P}<0.05)$ (Fig. 1B). The genes encoding lymphoid-lineage tyrosine kinases and costimulatory molecules (for example, TYRO3, SYK, CD79a and CD40 ligand), as well as those encoding molecules which were involved in the cell proliferation, hematopoiesis and leukemogenesis (for example, IRF8, TP53, WNT3, TGFbR2, BST2 and DDR3), were identified to share those pathways (Fig. 1B). In addition, the pathways of the immune effector process, immune response, and adaptive immune response, were determined to be enriched in the group of genes which had been deregulated in the Helios-1 overexpressed Jurkat cells (for example IL28b, APOBEC3C, TLR3, CCL1, CD7, and IL36). Furthermore, the overexpression of the zinc-finger transcription factor Helios-1 correlated with the pathways of the regulation of DNA binding and chromatin assembly [SUMO1, MSX2, histone cluster 1 (H1), H2, H3 and H4]. Also, this study detected and identified the differences between the Helios-1 and the control Jurkat cell lines in their expression of target genes, such as FOXN2 (1.363-fold), RUNX3 (1.142), MLLT4 (1.544), MAST3 (1.367), MSX2 (0.325), TP53 (0.513), CD79a (0.785), DNFIP2 (0.613), CD40LG (0.241), and SUMO1 (0.694), by utilizing quantitative PCR (Fig. 1C).

Modulation of the target gene expression by non-canonical short isoform Helios-4326-1431. The transcription profile regulated by the leukemic-type short isoform Helios- $\Delta 326-1431$ was evaluated through RNA analysis and microarray (Fig. 2A). Among the 940 genes ( $>1.5$-fold cut-off) which were dependent on Helios- $\Delta 326-1431$ for expression in the Jurkat lymphoblast cells, the changes were clustered in T-cell leukemogenesis and cell fate decisions (Table II). Several members, such as JUN (1.667-fold), MGMT (1.514), HRAS (1.963), TFPT (1.589), TAB1 (1.613), and KLF10 (0.643), were identified to be strong candidates for regulating T-lineage differentiation and involved in the development of leukemia. Other members showed the capacity to promote cell-fate decisions and morphogenesis, and were noted to be potential regulators of T-lineage commitment and homeostasis. Examples of these were HOXB7 (1.721-fold), GSC (2.036), HOXD11 (0.639), HES6 (1.646), NOTCH2NL (0.625), NKAP (2.059), and MSX2 (0.568). In addition, the modulators of the RAS signaling RAB40B (1.867-fold), RASSF7 (1.739), RASIP1 (1.683), and RASL10B (0.616) were deregulated as Helios- $\Delta 326-1431$ target genes. In addition, RNA polymerase and translation machinery factors, such as TAF1D (1.611-fold), POLR2G (1.644), EEF1G (2.173), TCEAL8 (1.666) and EIF1AY (1.610), were determined to be potential targets of the Helios- $\Delta 326-1431$.

Pathway analysis of the genes which were targeted by the non-canonical Helios- $\Delta 326-1431$ or by the wild-type Helios-1, provided insight into the functional consequences of the differences in the Helios isoforms which impact T-cell development and leukemogenesis $(\mathrm{P}<0.05)$. The genes which were associated with the Helios- $\Delta 326-1431$ showed considerable and specific enrichment of the pathways involved in cell growth, such as lymph node development (lymphotoxin), and the positive regulation of leukocyte proliferation (Vav3, SYK, IL23, CD24), as well as the cytokine-mediated signaling pathway (GBP1, IRF8, SUMO1, CCR8, EGR1, and CXCR3) (Fig. 2B). In addition, the lymphoid-specific genes which encoded the molecules involved in the positive regulation of leukocyte-mediated immunity, positive regulation of chronic inflammatory response, and Type-I interferon-mediated signaling pathway (ISG15, HLA-A, -C, and -E) were found to be specifically targeted by the Helios- $\Delta 326-1431$ gene. When compared with the full-length Helios-1, the non-canonical Helios- $\Delta 326-1431$ were associated with the translation, gene expression, and chromatin remodeling, such as the protein-DNA complex assembly (RPA2, RAD51 Recombinase, and $\mathrm{H} 1$ and $\mathrm{H} 2$ ), regulation of DNA binding (JUN, ID2, SUMO1, and HEY2), and chromatin assembly. These findings indicate that the non-canonical Helios- $\Delta 326-1431$ variant targeted the genetic networks that support lymphocyte differentiation and regulation of gene expressions. 
A

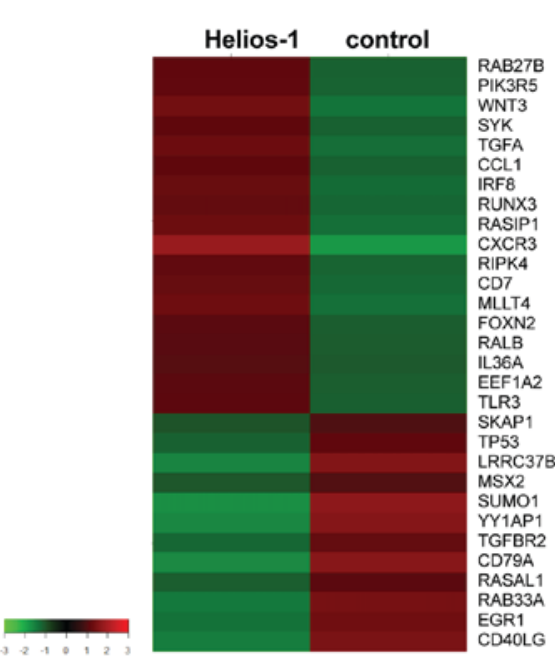

B

Significant Enriched GO Terms (Top 25)

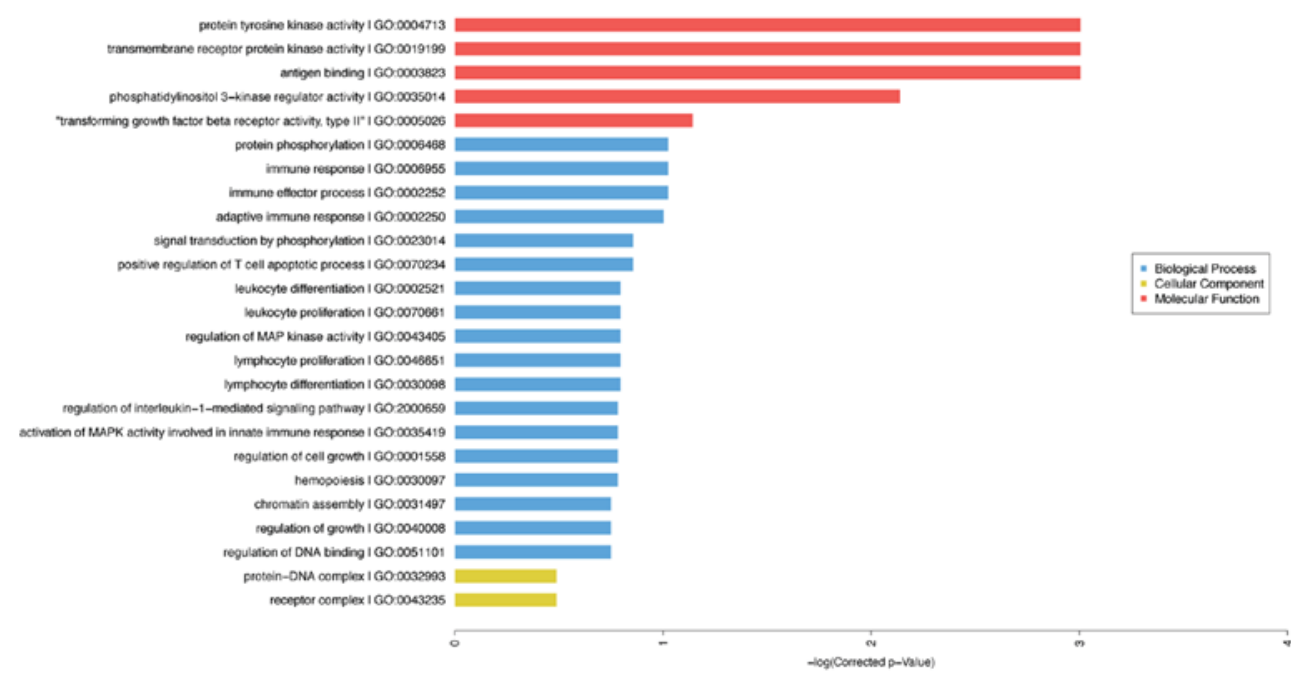

C

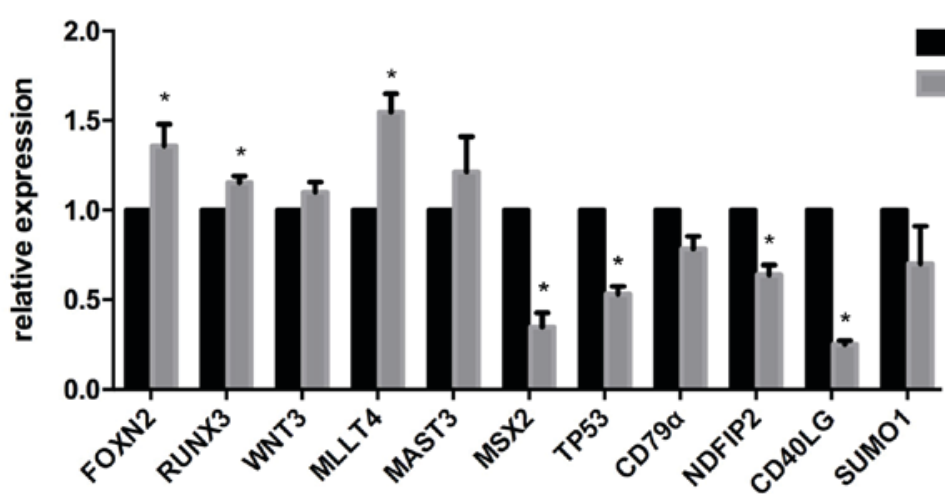

Figure 1. Comprehensive search for Helios-1 target genes which function in T-cell differentiation by microarray analysis. (A) The comparative expression analysis of the gene encoding molecules involved in the T-cell differentiation in overexpressed Helios-1, and control Jurkat cells, presented as a hierarchical clustering of the normalized expression. The Jurkat cells which stably expressed the Helios-1 and control were used to analyze the gene expressions using microarray. The 2D hierarchical clusters between the comparisons are indicated in the figure. (B) The pathway analysis of the genes deregulated in Helios-1 Jurkat $\mathrm{T}$ cells relative to their expression in the control cells. The P-values $(-\log )$ for the pathway discovery are indicated by the numbers along the vertical line. (C) Quantitative RT-PCR was used to analyze the relative gene expression in the Jurkat cells which stably expressed Helios-1, compared with the mock transfected Jurkat cells. Three independent experiments were performed, and asterisk (") indicates P<0.05 compared to control. Error bars represent SEM.

In order to examine the changes in the expression of the T-lineage transcription factors and regulatory genes during the Jurkat differentiation, we measured the transcript levels of the
Notch2NL, TCEAL8, ZNF593, NUDC, CBX8, HCST, TAB1, NKAP, TGFa, TNFSF9, MSX2, HOXD11, HEYL, and DGKQ in the Helios- $\Delta 326-1431$ overexpressed Jurkat cells, as well as 
Table I. T-cell target genes of Helios-1.

\begin{tabular}{|c|c|c|c|}
\hline Target gene & Location & $\begin{array}{l}\text { Expression during } \\
\text { T-cell specification }\end{array}$ & Function \\
\hline RAB27B & $18 \mathrm{q} 21.2$ & Upregulated & Small GTPase mediated signal transduction \\
\hline PIK3R5 & $17 \mathrm{p} 13.1$ & Upregulated & Cell growth, proliferation, differentiation, motility, survival and oncogenesis \\
\hline WNT3 & $17 q 21.32$ & Upregulated & Oncogenesis, regulation of cell fate and patterning, morphogenesis \\
\hline TGFA & $2 \mathrm{p} 13.3$ & Upregulated & Growth factor activity, angiogenesis and cancer \\
\hline CCL1 & $17 \mathrm{q} 12$ & Upregulated & Monocyte chemotaxis, immunoregulatory and inflammatory processes \\
\hline IRF8 & $16 q 24.1$ & Upregulated & Regulation of lineage commitment and in myeloid cell maturation \\
\hline RUNX3 & $1 \mathrm{p} 36.11$ & Upregulated & Transcription factor, and tumor suppressor, hematopoiesis \\
\hline RASIP1 & $19 q 13.33$ & Upregulated & Vascular-specific regulator of GTPase signaling, cell architecture, and adhesion \\
\hline CXCR3 & Xq13.1 & Upregulated & G protein-coupled receptor for chemokine, involved in leukocyte traffic \\
\hline RIPK4 & $21 \mathrm{q} 22.3$ & Upregulated & Serine/threonine protein kinase that interacts with PKC, activate NF- $\kappa \mathrm{B}$ \\
\hline CD7 & $17 q 25.3$ & Upregulated & Expressed on on thymocytes and mature $\mathrm{T}$ cells, play a role in $\mathrm{T}$-cell interactions \\
\hline MLLT4 & $6 q 27$ & Upregulated & Involved in acute myeloid leukemias with $\mathrm{t}(6 ; 11)(\mathrm{q} 27 ; \mathrm{q} 23)$ translocation \\
\hline FOXN2 & $2 \mathrm{p} 16.3$ & Upregulated & Transcription factor activity, T-Cell leukemia \\
\hline RALB & $2 q 14.2$ & Upregulated & Pathways in cancer and signaling by GPCR \\
\hline IL36A & $2 q 14.1$ & Upregulated & Activate $\mathrm{NF}-\kappa \mathrm{B}$ and MAPK signaling pathways in inflammatory response \\
\hline EEF1A2 & $20 q 13.33$ & Upregulated & Protein biosynthesis, enzymatic delivery of aminoacyl tRNAs to the ribosome \\
\hline TP53 & 17 p13.1 & Downregulated & Cell cycle arrest, apoptosis, senescence, DNA repair, and tumor formation \\
\hline MSX2 & $5 q 35.2$ & Downregulated & Transcription factor activity, development \\
\hline TGFBR2 & $3 \mathrm{p} 24.1$ & Downregulated & TGF- $\beta$ signaling pathway \\
\hline CD40LG & $\mathrm{Xq} 26.3$ & Downregulated & T-cell immune responses, T-cell proliferation and cytokine production \\
\hline
\end{tabular}

RAB27B, member RAS oncogene family; PIK3R5, phosphoinositide-3-kinase regulatory subunit 5; wnt3, wnt family member 3; TGFA, transforming growth factor $\alpha$; CCL1, C-C motif chemokine ligand 1; IRF8, interferon regulatory factor 8; RUNX3, runt related transcription factor 3; RASIP1, ras interacting protein 1; CXCR3, C-X-C motif chemokine receptor 3; RIPK4, receptor interacting serine/threonine kinase 4; FOXN2, forkhead box N2; RALB, RAS like proto-oncogene B; IL36A, interleukin 36, $\alpha$; EEF1A2, eukaryotic translation elongation factor $1 \alpha 2$; TP53, tumor protein P53; MSX2, Msh homeobox 2; TGFBR2, transforming growth factor $\beta$ receptor 2; CD40LG, CD40 ligand.

the control cells, using real-time PCR (Fig. 2C). The results were consistent with microarray data, and showed significant upregulations of the TCEAL8 (1.462-fold), ZNF593 (1.699), CBX8 (1.425), TAB1 (1.761), NKAP (1.558), TGFa (1.309), and TNFSF9 (3.673). In contrast, downregulations were observed in the expressions of the HOXD11 (0.103), HEYL (0.111), and DGKQ (0.082).

Surface marker expression. In order to evaluate the role of the Helios isoforms in the differentiation of the T lymphoblasts (17), the expressions of the CD4, CD8, CD3, CD25, and CD7 on the cell surface of Jurkat cells were tested on the 30th day after the transduction by flow cytometry (Fig. 3). The results showed that the cells which expressed CD3 at day 30 were decreased in the Helios- $\Delta 326-1431$ transduced cells $(47.6 \pm 3 \%)$ when compared with the groups of the Helios-1 transduced cells $(59.0 \pm 4 \%)$, or the control $(57.6 \pm 3 \%)$ (Fig. 3A). The CD25 expression was determined to be increased in the Helios- $\Delta 326-1431$ cells $(3.59 \pm 0.5 \%)$ when compared to the Helios-1 $(0.59 \pm 0.3 \%)$ or control cell groups $(0.33 \pm 0.2 \%)$ (Fig. $3 \mathrm{C})$. However, there was no difference between Helios-1 and control for CD3 and CD25 expression (Fig. 3). This is consistent with the report that Helios functions as a master transcription factor in $\mathrm{CD}^{+}$ $\mathrm{CD}_{25} 5^{+}$regulatory $\mathrm{T}$ cells $(9,18)$. The expression patterns of
CD7, which plays a role in T-cell interactions (19), were not changed upon the stable overexpression of Helios isoforms (Fig. 3D). Therefore, the non-canonical Helios- $\Delta 326-1431$ isoform regulates the cell surface expression of the T-lineage markers.

\section{Discussion}

In this study, the effects of the alternative splicing variants of Helios on the differentiation of the Jurkat T lymphoblast cell line were evaluated. Also, the data of mRNA microarray were assessed, and the deregulated expression of the vital regulatory gene involved in the T-cell proliferation, differentiation, and leukemic transformation was confirmed. This led to the identification of the gene profiles which were controlled by the full-length Helios-1, as well as the non-canonical leukemic-type short isoform Helios- $\Delta 326-1431$.

The presence of the alternative splice Helios variants was first revealed in research studies utilizing the primary leukemic cells from patients with newly diagnosed ALL (12-14). In this study, we observed that dominant-negative Helios- $\Delta 326-1431$ potentially induces the expression of several oncogenes. According to the results, the Helios- $\Delta 326-1431$ significantly induced the upregulation of the molecules, such as the JUN proto-oncogene, which may be involved in the leukemogenesis 


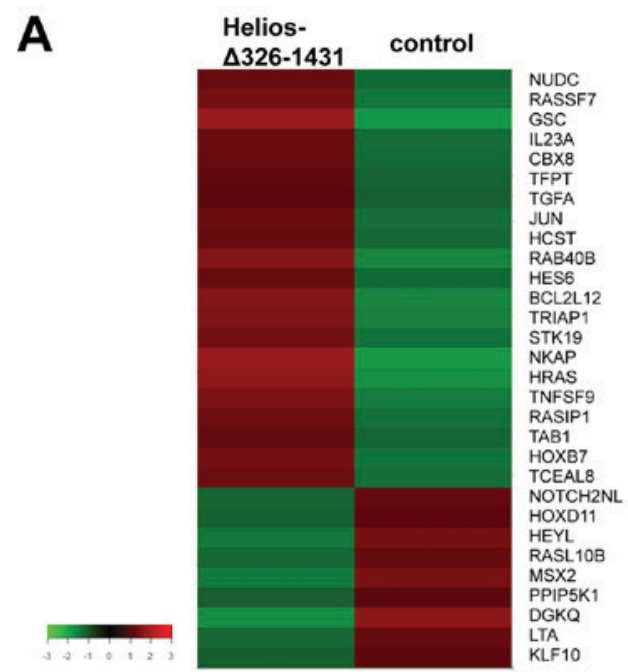

B

Significant Enriched GO Terms (Top 30)
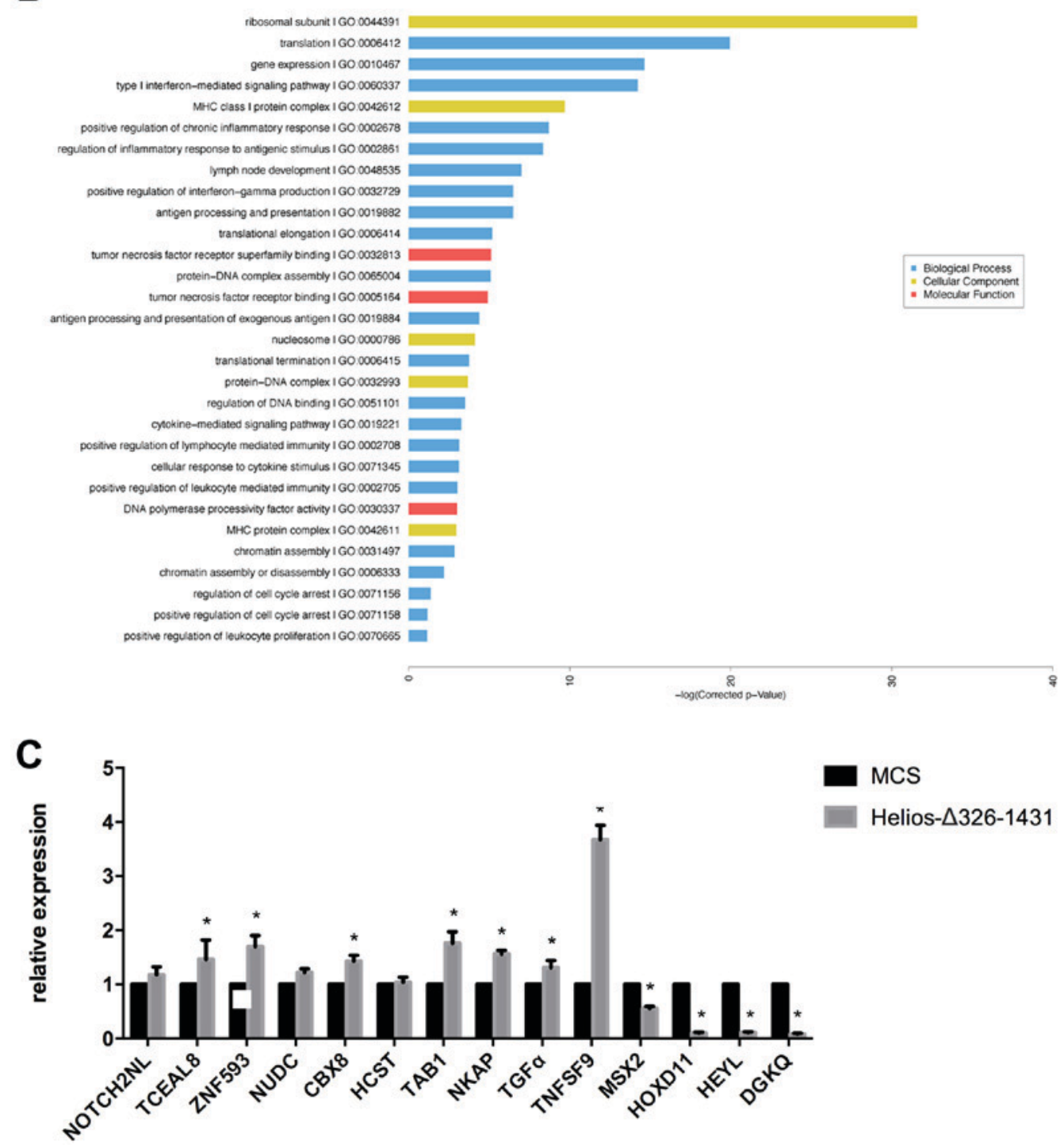

Figure 2. Expression analysis of the genes targeted by the Helios- $\Delta 326-1431$. (A) The gene expression analysis and clustering of the genes in the Helios- $\Delta 326-1431$ transduced Jurkat cells compared to the control. The gene expression patterns of the Jurkat cells which expressed Helios- $\Delta 326-1431$ were comprehensively analyzed using the microarray technique. The obtained $2 \mathrm{D}$ hierarchical cluster between the cells which did or did not express the Helios- $\Delta 326-1431$ is shown. (B) The gene ontology of the deregulated genes targeted by Helios- $\Delta 326-1431$, which was established in the Jurkat cells transduced with the non-canonical or wild-type Helios isoforms. (C) The quantitative-polymerase chain reaction of the Helios- $\Delta 326-1431$ target genes from the mRNAs which were isolated from the transduced Jurkat cells, and the control. Three independent experiments were performed, and asterisk ( $\left(^{*}\right)$ indicates $\mathrm{P}<0.05$ compared to control. Error bars represent SEM. 
Table II. T-cell target genes of Helios- $\Delta 326-1431$.

\begin{tabular}{lcll}
\hline $\begin{array}{l}\text { Target } \\
\text { gene }\end{array}$ & Location & $\begin{array}{c}\text { Expression } \\
\text { during T-cell } \\
\text { specification }\end{array}$ & \\
\hline GSC & $14 q 32.13$ & Upregulated & Act as a transcription factor in the development during embryogenesis \\
IL23A & $12 \mathrm{q} 13.3$ & Upregulated & Stimulate the production of IFN- $\gamma$, act on memory CD4(+) T cells \\
CBX8 & $17 \mathrm{q} 25.3$ & Upregulated & Transcriptional repressor, chromatin remodeling \\
TFPT & $19 \mathrm{q} 13.42$ & Upregulated & Associated with childhood leukemia \\
TGFA & $2 \mathrm{p} 13.3$ & Upregulated & Activate a signaling pathway for cell proliferation, differentiation and development \\
JUN & $1 \mathrm{p} 32.1$ & Upregulated & Sequence-specific DNA binding to regulate gene expression, involved in malignancies \\
HCST & $19 \mathrm{q} 13.12$ & Upregulated & Activate PI3K dependent signaling pathways, cell survival and proliferation \\
HES6 & $2 \mathrm{q} 37.3$ & Upregulated & Regulate cell differentiation, participate in Notch-mediated HES/HEY network \\
NKAP & Xq24 & Upregulated & Activation of the ubiquitous transcription factor NF- $\mathrm{kB}$ \\
HRAS & $11 \mathrm{p} 15.5$ & Upregulated & Signal pathways by intrinsic GTPase activity, involved in development, cancer \\
TNFSF9 & $19 \mathrm{p} 13.3$ & Upregulated & Involved in the antigen presentation process and in the generation of cytotoxic T cells \\
HOXB7 & $17 \mathrm{q} 21.32$ & Upregulated & Transcription factor that is involved in cell proliferation, differentiation and development \\
HEYL & $1 \mathrm{p} 34.2$ & Downregulated & An effector of Notch signaling and a regulator of cell fate decisions \\
PPIP5K1 & $15 \mathrm{q} 15.3$ & Downregulated & Intracellular signaling pathways of Inositol phosphates \\
LTA & $6 \mathrm{p} 21.33$ & Downregulated & Mediate a large variety of inflammatory, immunostimulatory, and antiviral responses \\
KLF10 & $8 \mathrm{q} 22.3$ & Downregulated & Transcriptional repressor, inhibitory activity of cancer growth \\
\hline
\end{tabular}

GSC, goosecoid homeobox; IL23A, interleukin 23 subunit $\alpha$; CBX8, chromobox 8; TFPT, TCF3 (E2A) fusion partner; TGFA, transforming growth factor $\alpha$; JUN, jun proto-oncogene; HCST, hematopoietic cell signal transducer; HES6, hes family BHLH transcription factor 6; NKAP, NF- $\mathrm{KB}$ activating protein; HRAS, HRas proto-oncogene, GTPase; TNFSF9, tumor necrosis factor superfamily member 9; HOXB7, homeobox B7; HEYL, hairy/enhancer-of-split related with YRPW motif 3; PPIP5K1, diphosphoinositol pentakisphosphate kinase 1; LTA, lymphotoxin $\alpha$; KLF10, Kruppel like factor 10 .

of Helios-deregulated T-ALL (20). In addition, we observed that ectopic expression of Helios- $\Delta 326-1431$ led to the upregulated expression of HRAS, which exhibited leukemogenic potential in myeloid-lineage leukemia (21). Furthermore, this study identified that other components of the RAS signaling pathway, such as RASSF7, RAB40B, and RASIP1, were upregulated in the Helios- $\Delta 326-1431$ overexpressed cells Interestingly, TFPT [TCF3 (E2A) fusion partner], which has been implicated in childhood leukemia (22), was also found to be upregulated in the Helios- $\Delta 326-1431$ cells. These targeted genes consolidated the activation of the programs which promoted survival and cell proliferation, even though their deregulation led to leukemic transformation. The overexpression of non-canonical Helios may provide aberrant survival properties to the differentiating thymocytes, which are predisposed for further selection in the activations of oncogene mutations and malignant phenotypes. These analyses from the microarray data indicate that the non-canonical short Helios isoform functions as an oncogenic variant and initiates leukemogenesis.

This study also identified that the genes which encode the T-lineage cell markers and chemotaxis molecules, such as CD7, CCL1, and CXCR3, are upregulated in the wild-type Helios-1 overexpressed Jurkat cells, whereas the B-lineage cell marker CD79a are downregulated. It has been reported that regulatory $\mathrm{T}$-cells initiate recruitment and suppressive function via a CCL1 dependent pathway $(23,24)$. In addition, the CXCR3 signaling directly induces the mobilization and recruitment of Tregs (25). Therefore, the Helios-1 may function as the transcription factor for regulatory T-cells by targeting the CCL1 and CXCR3 molecules. The microarray data also confirmed that the tyrosine kinases, TAM family receptor tyrosine kinase TYRO3 and SYK, are upregulated in the Helios-1 overexpressed Jurkat T-cells. The TYRO3 functions as a negative regulator of type 2 immunity (26), whereas the SYK has the ability to modulate the $\mathrm{CD}^{+} \mathrm{T}$ cell response (27). The GO analysis indicates that the pathways of the immune effector process and immune response (for example IL28, IL36, and TLR3) are enriched in the Helios-1 $\mathrm{T}$ cells. The results of the research by Asanuma et al were in agreement with this finding, and confirmed that the Helios variants regulated the pathway of the TNF receptor binding (11). Therefore, consistent with previous data, these deregulated patterns of gene expression suggest that wild-type Helios-1 has an immunomodulatory role in T-cell development.

It is interesting to note that the results of the previous research demonstrated that the dominant-negative isoform of Ikaros, Ik-6 was overexpressed in patients with blast crisis of chronic myelogenous leukemia, and acute B-lymphoblastic leukemia (28). These findings suggest that Ikaros plays the role of a tumor suppressor gene in the myeloid and B-cell lineages (28). In support of this viewpoint, Mullighan et al defined the oncogenic lesions that cooperate with $B C R-A B L 1$ to induce ALL by genome-wide analysis, and identified that Ikaros was deleted in $83.7 \%$ of BCR-ABL1 lymphoblastic leukemia (29). In contrast, the T-cell malignancies were only observed in the gene-targeted mice of Ikaros (30). The related 
A

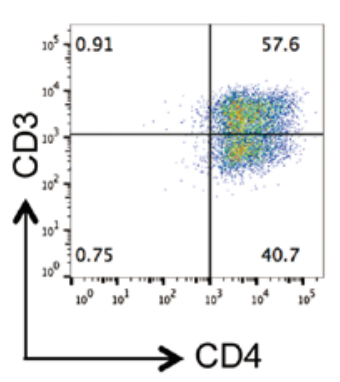

Helios-1

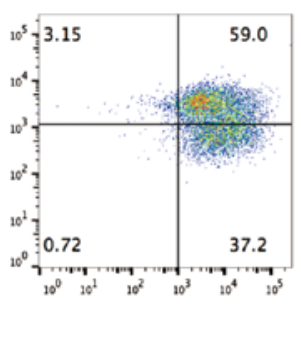

B
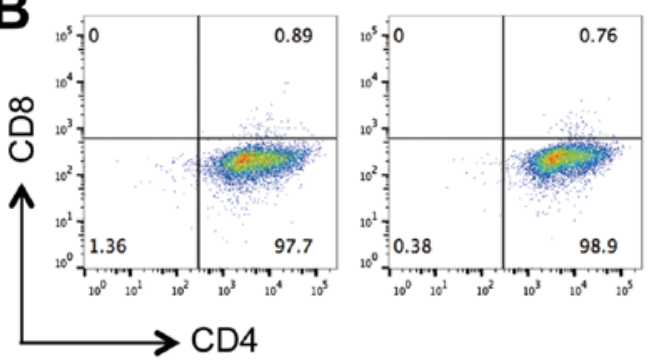

C
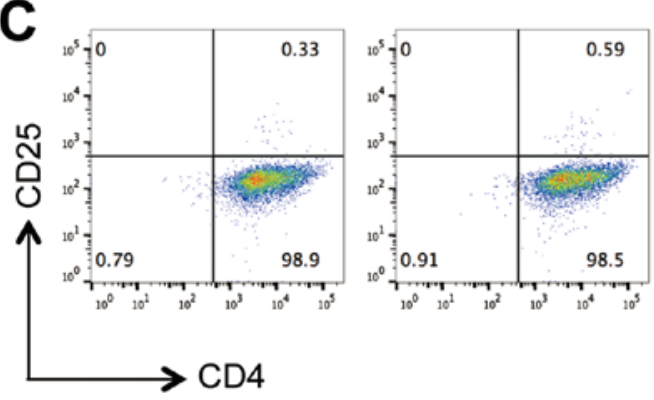

D
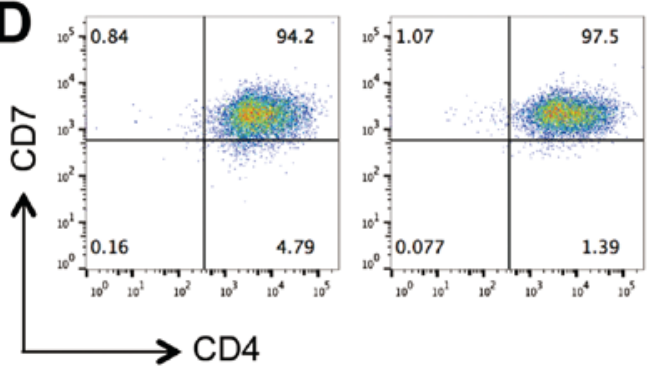
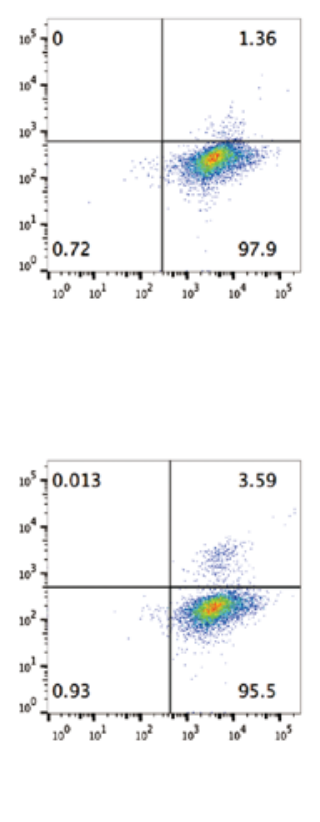

Helios- $\Delta 326-1431$
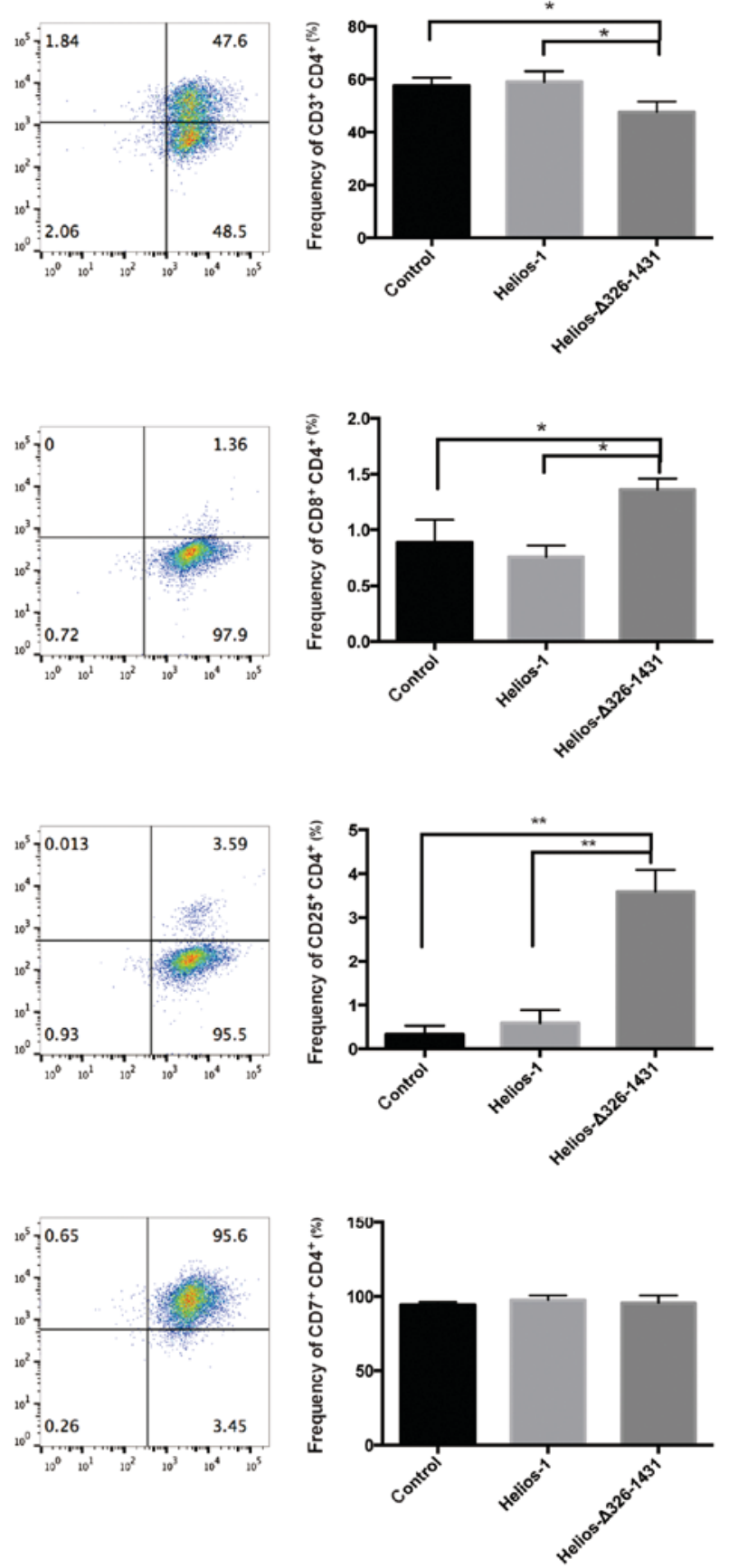

Figure 3. Expression analysis of the surface markers CD3, CD8, CD25, and CD7 using flow cytometry. The profiles showed the expression of the CD molecules in the groups transduced by Helios-1, Helios- $\Delta 326-1431$, or the backbone of the vector. The top panel (A) shows the CD3 and CD4 staining. The middle two (B and C) are the CD8 and CD25 staining with CD4, and the bottom panel (D) shows the expression of CD7 and CD4 in total Jurkat cells which had been transduced with the Helios variants. The numbers indicate the percentage of cells in each of the subsets. The right panel indicates the quantification of the cells in FACS profiles. Data shown are the mean percentage \pm standard deviation $(n=3)$. ${ }^{*} \mathrm{P}<0.05$ and ${ }^{* * *} \mathrm{P}<0.01$.

gene Helios has been shown to frequently exhibit multiple dominant-negative isoforms in patients with T-cell acute leukemia (6,11-14). In the mouse model, the overexpression of the artificial dominant-negative Helios isoform leads to increased T-cell proliferation, as well as the development of T-cell lymphomas (31). However, in Helios-deficient mouse line, Helios is not essential for the development, homeostasis, and function of the thymic-derived $\mathrm{T}$ lymphocytes, which suggests that other Ikaros family members possibly compensate for the Helios in the T-cells (32). Overall, these data indicate that the coordinated deregulation of the Ikaros gene family may lead to human hematologic malignancies.

In this study, the roles of Helios in target gene activations and repressions, as well as T-cell development, were investigated. The findings in this study suggest that the full-length Helios-1 may play a decisive role in shaping the Treg cell identity. In addition, Helios family members are thought to coordinate gene transcription through chromatin remodeling, and previous research showed that Ikaros and Helios interact in the nucleosome remodeling complex of DNA-dependent ATPase Mi-2 and histone 
deacetylases $(6,33)$. Thus, it is possible that Helios-1 modulates the expression of CD25, which is the marker for distinguishing Treg cells (Fig. 3C). In contrast, the proportion of CD25+ cells was increased in the Jurkat $\mathrm{T}$ lymphoblast cells that were stably transduced with dominant-negative Helios- $\Delta 326-1431$. Therefore, the dominant-negative Helios- $\Delta 326-1431$ isoform promotes the leukemogenesis and T-cell differentiation by inhibiting the activity of the functional Ikaros proteins, and also defining the leukemic transcriptional program, such as HRAS, TGF, and JUN.

The events initiated by Helios overexpression consolidate activation of the gene expression programs, which promote cell growth and survival. When deregulated, this process gives rise to leukemic transformation. The alternative splicings of Helios isoforms are part of a regulatory mechanism, which is effective during T-cell development and leukemogenesis. Helios also controls a network of epigenetic and transcriptional regulators during the normal T-cell development and leukemogenesis. Therefore, targeting the transcriptional regulation of Helios may open new avenues for leukemia treatment.

\section{Acknowledgements}

The authors would like to thank Dr. Alan Holt and Dr. Phillip Bryant for their assistance in editing this manuscript.

\section{Funding}

This study was supported by grants from National Science Foundation (grant no. NSF 81202309; nsfc.gov.cn/), Tianjin City Foundation of Basic and Advanced Research (grant no. 13JCQNJC11200; tstc.gov.cn/) and the New Teacher Fund from the Ministry of Education (grant no. 20120031120057) to FL. The funders had no role in study design, data collection and analysis, decision to publish, or preparation of the manuscript.

\section{Availability of data and materials}

All data generated or analyzed during this study are included in this published article.

\section{Authors' contributions}

YIL, YAL and CL performed experiments and analyzed the data. FL designed research, analyzed data and wrote the paper. FL, JL and DL performed experiments and provided technical support. WZ and WL assisted with the experiments.

\section{Ethics approval and consent to participate}

Not applicable.

\section{Consent for publication}

Not applicable.

\section{Competing interests}

The authors declare that they have no competing interests.

\section{References}

1. Yui MA and Rothenberg EV: Developmental gene networks: A triathlon on the course to T cell identity. Nat Rev Immunol 14: 529-545, 2014.

2. Gómez-del Arco P, Kashiwagi M, Jackson AF, Naito T, Zhang J, Liu F, Kee B, Vooijs M, Radtke F, Redondo JM and Georgopoulos K: Alternative promoter usage at the Notch1 locus supports ligand-independent signaling in T cell development and leukemogenesis. Immunity 33: 685-698, 2010.

3. McCormack MP, Shields BJ, Jackson JT, Nasa C, Shi W, Slater NJ, Tremblay CS, Rabbitts TH and Curtis DJ: Requirement for Lyl1 in a model of Lmo2-driven early T-cell precursor ALL. Blood 122: 2093-2103, 2013.

4. Kelley CM, Ikeda T, Koipally J, Avitahl N, Wu L, Georgopoulos K and Morgan BA: Helios, a novel dimerization partner of Ikaros expressed in the earliest hematopoietic progenitors. Curr Biol 8: 508-515, 1998.

5. Kim HJ, Barnitz RA, Kreslavsky T, Brown FD, Moffett H, Lemieux ME, Kaygusuz Y, Meissner T, Holderried TA, Chan S, et al: Stable inhibitory activity of regulatory $\mathrm{T}$ cells requires the transcription factor Helios. Science 350: 334-339, 2015.

6. Zhao S, Liu W, Li Y, Liu P, Li S, Dou D, Wang Y, Yang R, Xiang R and Liu F: Alternative splice variants modulates dominant-negative function of Helios in T-Cell leukemia. PLoS One 11: e0163328, 2016.

7. Fontenot JD, Rasmussen JP, Gavin MA and Rudensky AY: A function for interleukin 2 in Foxp3-expressing regulatory T cells. Nat Immunol 6: 1142-1151, 2005.

8. Hill JA, Feuerer M, Tash K, Haxhinasto S, Perez J, Melamed R, Mathis D and Benoist C: Foxp3 transcription-factor-dependent and -independent regulation of the regulatory $\mathrm{T}$ cell transcriptional signature. Immunity 27: 786-800, 2007.

9. Getnet D, Grosso JF, Goldberg MV, Harris TJ, Yen HR, Bruno TC, Durham NM, Hipkiss EL, Pyle KJ, Wada S, et al: A role for the transcription factor Helios in human CD4(+)CD25(+) regulatory T cells. Mol Immunol 47: 1595-1600, 2010.

10. Sridharan R and Smale ST: Predominant interaction of both Ikaros and Helios with the NuRD complex in immature thymocytes. J Biol Chem 282: 30227-30238, 2007.

11. Asanuma S, Yamagishi M, Kawanami K, Nakano K, Sato-Otsubo A, Muto S, Sanada M, Yamochi T, Kobayashi S, Utsunomiya A, et al: Adult T-cell leukemia cells are characterized by abnormalities of Helios expression that promote $\mathrm{T}$ cell growth. Cancer Sci 104: 1097-1106, 2013.

12. Nakase K, Ishimaru F, Fujii K, Tabayashi T, Kozuka T, Sezaki N, Matsuo Y and Harada M: Overexpression of novel short isoforms of Helios in a patient with T-cell acute lymphoblastic leukemia. Exp Hematol 30: 313-317, 2002.

13. Fujii K, Ishimaru F, Nakase K, Tabayashi T, Kozuka T, Naoki K, Miyahara M, Toki H, Kitajima K, Harada M and Tanimoto M: Over-expression of short isoforms of Helios in patients with adult T-cell leukaemia/lymphoma. Br J Haematol 120: 986-989, 2003.

14. Tabayashi T, Ishimaru F, Takata M, Kataoka I, Nakase K, Kozuka T and Tanimoto M: Characterization of the short isoform of Helios overexpressed in patients with T-cell malignancies. Cancer Sci 98: 182-188, 2007.

15. Landgraf P, Rusu M, Sheridan R, Sewer A, Iovino N, Aravin A, Pfeffer S, Rice A, Kamphorst AO, Landthaler M, et al: A mammalian microRNA expression atlas based on small RNA library sequencing. Cell 129: 1401-1414, 2007.

16. Joshi I, Yoshida T, Jena N, Qi X, Zhang J, Van Etten RA and Georgopoulos K: Loss of Ikaros DNA-binding function confers integrin-dependent survival on pre-B cells and progression to acute lymphoblastic leukemia. Nat Immunol 15: 294-304, 2014

17. Saki N, Abroun S, Soleimani M, Mortazavi Y, Kaviani S and Arefian E: The roles of miR-146a in the differentiation of Jurkat T-lymphoblasts. Hematology 19: 141-147, 2014.

18. Thornton AM, Korty PE, Tran DQ, Wohlfert EA, Murray PE, Belkaid Y and Shevach EM: Expression of Helios, an Ikaros transcription factor family member, differentiates thymic-derived from peripherally induced Foxp $3+\mathrm{T}$ regulatory cells. J Immunol 184: 3433-3441, 2010.

19. Mitchell JL, Seng A and Yankee TM: Ikaros, Helios, and Aiolos protein levels increase in human thymocytes after $\beta$ selection. Immunol Res 64: 565-575, 2016. 
20. Kollmann K, Heller G, Ott RG, Scheicher R, Zebedin-Brandl E, Schneckenleithner C, Simma O, Warsch W, Eckelhart E, Hoelbl A, et al: c-JUN promotes BCR-ABL-induced lymphoid leukemia by inhibiting methylation of the 5' region of Cdk6. Blood 117: 4065-4075, 2011.

21. Parikh C, Subrahmanyam R and Ren R: Oncogenic NRAS, KRAS, and HRAS exhibit different leukemogenic potentials in mice. Cancer Res 67: 7139-7146, 2007.

22. Brambillasca F, Mosna G, Ballabio E, Biondi A, Boulukos KE and Privitera E: Promoter analysis of TFPT (FB1), a molecular partner of TCF3 (E2A) in childhood acute lymphoblastic leukemia. Biochem Biophys Res Commun 288: 1250-1257, 2001.

23. Mira E, León B, Barber DF, Jiménez-Baranda S, Goya I, Almonacid L, Márquez G, Zaballos A, Martínez-A C, Stein JV, et al: Statins induce regulatory $\mathrm{T}$ cell recruitment via a CCL1 dependent pathway. J Immunol 181: 3524-3534, 2008.

24. Hoelzinger DB, Smith SE, Mirza N, Dominguez AL, Manrique SZ and Lustgarten $\mathrm{J}$ : Blockade of CCL1 inhibits $\mathrm{T}$ regulatory cell suppressive function enhancing tumor immunity without affecting T effector responses. J Immunol 184: 6833-6842, 2010

25. Li CX, Ling CC, Shao Y, Xu A, Li XC, Ng KT, Liu XB, Ma YY, Qi X, Liu H, et al: CXCL10/CXCR3 signaling mobilized-regulatory $\mathrm{T}$ cells promote liver tumor recurrence after transplantation. J Hepatol 65: 944-952, 2016.

26. Chan PY, Carrera Silva EA, De Kouchkovsky D, Joannas LD, Hao L, Hu D, Huntsman S, Eng C, Licona-Limón P, Weinstein JS, et al: The TAM family receptor tyrosine kinase TYRO3 is a negative regulator of type 2 immunity. Science 352 99-103, 2016.
27. Chauhan AK, Moore TL, Bi Y and Chen C: Fc $\gamma$ RIIIa-Syk Co-signal modulates CD4+ T-cell response and Up-regulates Toll-like Receptor (TLR) expression. J Biol Chem 291: 1368-1386, 2016.

28. Beer PA, Knapp DJ, Miller PH, Kannan N, Sloma I, Heel K, Babovic S, Bulaeva E, Rabu G, Terry J, et al: Disruption of IKAROS activity in primitive chronic-phase CML cells mimics myeloid disease progression. Blood 125: 504-515, 2015.

29. Mullighan CG, Miller CB, Radtke I, Phillips LA, Dalton J, Ma J, White D, Hughes TP, Le Beau MM, Pui CH, et al: BCR-ABL1 lymphoblastic leukaemia is characterized by the deletion of Ikaros. Nature 453: 110-114, 2008.

30. Winandy S, Wu P and Georgopoulos K: A dominant mutation in the Ikaros gene leads to rapid development of leukemia and lymphoma. Cell 83: 289-299, 1995.

31. Zhang Z, Swindle CS, Bates JT, Ko R, Cotta CV and Klug CA: Expression of a non-DNA-binding isoform of Helios induces T-cell lymphoma in mice. Blood 109: 2190-2197, 2007.

32. Cai Q, Dierich A, Oulad-Abdelghani M, Chan S and Kastner P: Helios deficiency has minimal impact on $\mathrm{T}$ cell development and function. J Immunol 183: 2303-2311, 2009.

33. Kim J, Sif S, Jones B, Jackson A, Koipally J, Heller E, Winandy S, Viel A, Sawyer A, Ikeda T, et al: Ikaros DNA-binding proteins direct formation of chromatin remodeling complexes in lymphocytes. Immunity 10: 345-355, 1999. International (CC BY-NC-ND 4.0) License. 